$c^{\wedge}$

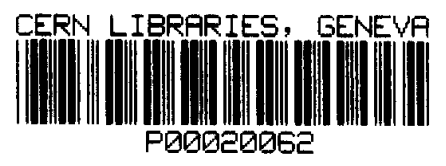

CERN AT/93-50 (IC)
SW 9403

\title{
Integrating a Commercial Industrial Control System to the Accelerator Control System: A Case Study
}

F. Momal, J. Brahy, R. Saban and P. Sollander 


\title{
INTEGRATING A COMMERCIAL INDUSTRIAL CONTROL SYSTEM TO THE ACCELERATOR CONTROL SYSTEM: A CASE STUDY
}

\author{
F. Momal, J. Brahy, R. Saban, P. Sollander \\ CERN, Geneva, Switzerland
}

At CERN a large number of systems providing services (cooling and ventilation, cryogenics, electricity distribution, personnel and building safety, etc.) are controlled by industrial PLCs. A commercial supervision package, providing local supervision facilities is used as a gateway to the accelerator control system. The integration of such a system in the CERN accelerator control environment addresses such issues as connections to control-rooms and desktop computers, alarms logging, remote or alien man-machine interfaces. The paper describes the components of the system and reports the first operational experience.

\section{Introduction}

Recent developments have shown an increasing number of utilities at CERN controlled by industrial systems. The process control solutions nowadays offered by industry essentially fulfil our needs. These industrial control systems can be divided in two categories: the DCS (Distributed Control System) and the PLC (Programmable Logic Controller). Classical examples of utilities using such systems at CERN are cooling and ventilation, cryogenics, electric distribution, personnel and building safety. The system entities (PLC) communicate on a local field-bus generally via proprietary protocols. Supervisory consoles hierarchically higher in the CIM (Computer Integrated Manufacturing) architecture follow and supervise the process. The control system includes the different layers going from the PLC up to the supervisor.

The way we have solved the integration's problems caused by using such systems at CERN will be illustrated by a precise example: the integration of FactoryLink=, a supervisory system from USDATA. This supervisor is mainly used in conjunction with Siemens PLCs to control service equipment already installed or planned for the near future: access control to radiation zone in the SPS accelerator, fire detection systems, ... We will present the building safety system of new experimental areas.

\section{The Process and its Control}

\subsection{The Building Safety System}

The building safety system on which we have installed an industrial control system includes numerous processes: gas detection systems, fire detection systems, electrical supply distribution and ventilation.

The total amount of I/O links from the process to the control system is in the order of 150 analog inputs, 280 digital inputs and 30 digital outputs per zone.

\subsection{The Control Architecture}

Fire detection sensors give digital signals which have to be collected and processed. This is done by Siemens PLCs(Programmable Logic Controller), the market leader in Europe. These Siemens PLCs also gather data coming from the gas detection systems and control the electrical supply and the ventilation by means of digital output cards (see fig. 1).

PLCs communicate on local fieldbuses generally via proprietary protocols. SiemensPLCs are able to communicate on different kind of networks. The SINECL2 bus satisfied the operational requirements (speed, distance) and proved to be economical since we could use the already installed cable infrastructure. In other new installations we chose SINEC H1 on Ethernet for performance and compatibility.

To complete the control system such issues as graphical supervision, alarm reporting and archiving have to be addressed. The use of the standard elements of the CERN accelerators controls would lead to an increasing number of software layers and would force us to develop drivers for each industrial proprietary protocol. We preferred an industrial supervisory system which completeness includes all types of PLC protocol drivers and all required services (alarm management, animated graphics).

\subsection{The Supervisory System}

The accelerator controls at CERN are based on the UNDX operating system. For compatibility reasons we had to look for a supervisor which runs on UNIX computers. We have selected FactoryLink- from USDATA for its completeness and its advantage of running on multiple platforms (HP-UX, AIX, MS-Windows, OS2, VMS). For this specific implementation we use HP 9000 machines running HP-UX. Like most of the supervisory systems FactoryLink= consists of:

- acentral database containing data relevant to the controlled equipment. This database is real-time in the sense that it is continuously refreshed. It contains for example, analog inputs/outputs from some PLCs, calculated values of some physical parameters, initialisation values.

- some external modules which communicate with the central database: PLCs' protocol drivers, man-machine interfaces, report generators, ...

FactoryLink comes with an application generator which provides the user with all the tools needed to define a process control application:

- animated man/machine interface via synoptics, trend curves and control panels

- process sequencing using recipes

- database logging and archiving

- alarm management

A local supervision application is dedicated to each previously described process.

${ }^{1}$ The gaz detection systems are intelligent systems which communicate via an industrial protocol called JBUS. 


\section{Integration Issues}

In order to be able to use a commercial industrial control system at CERN, some specific requirements must be met. They are to be integrated in the existing network structure. They must provide data to the central services which are the control rooms and the Central Alarm Server (CAS), and have to be remotely accessible over large distances.

\subsection{Network Structure}

Constraints brought by the industrial control systems (realtime, proprietary protocol, heavy network load) result in a necessary full independence between field-buses and their CERN peers. Therefore the industrial control system and the CERN control system were linked at the level of the supervisor acting in addition as a gateway. The two networks are totally distinct and the data exchange between them is organised in such a way that only relevant data are sent to the control room. The local RS485 network is used for the communications between FactoryLink- and the Siemens PLCs using the Siemens L2 protocol while the CERN general Ethernet network allows to communicate remotely with TCP/IP (see fig. 1). mailboxes while the access to the database is mainly eventdriven. For example an alarm module will subscribe to a value uploaded from a given sensor. When this valuechanges, the module will be called and will then check whether this value lies within defined limits.

In order to ease the portability of our developments to other supervisors, we developed an intermediate layer in the form of a library. This library offers such routines as:

- read_tag_on_change 0 : subscribe to a database object and wait for a change on this object

- write_tag0 : modify the value of a database object - read_alarm0 : read the active alarms related to the supervised process

Porting the modules acting for the integration or any other facility developed to increase the functionality, necessitates the rewrite of the library and the recompilation of the $C$ modules. This exercise has been done on an additional system used for the control of the LEP200 cryogenics (ABBSuperviewm).

\subsection{Connection with the Central Services}

Using this standard library we have developed $\mathrm{C}$ modules to

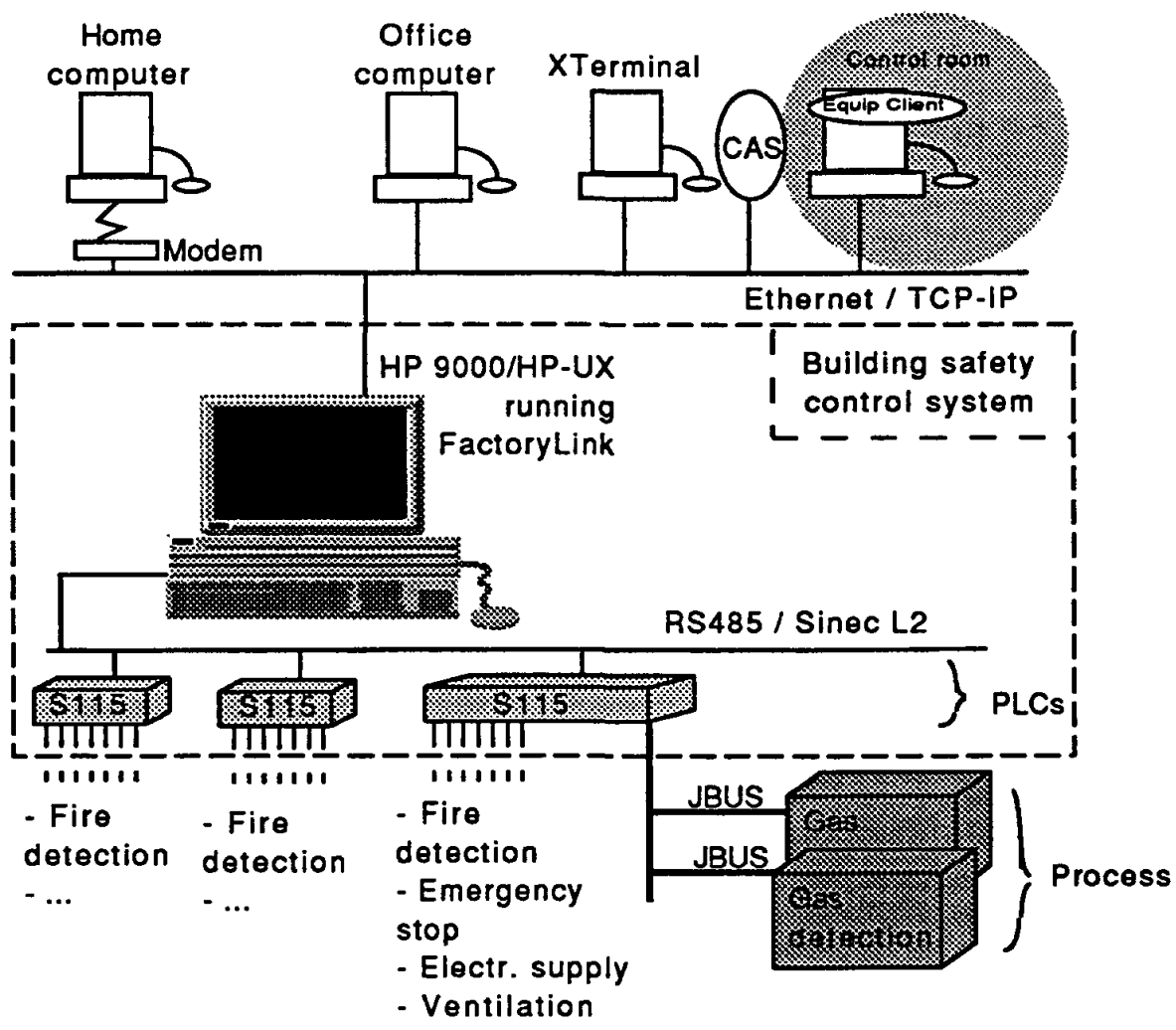

Figure 1

Figure 1: The building safety control system and its integration at CERN

\subsection{Standardising Software Development}

Software integration is achieved by implementing additional tools at the supervisory level (in the HP9000 computer). The selected supervisor (FactoryLink-) allows developers to add new external modules linked to the central real-time database. These new modules are written in C and use a detailed API (application programming interface) to interact with the central real-time database and with the other existing modules. Communication between modules is made by the mean of provide data to the central services.

- The accelerator control group uses a CERN developed portable communication package based on Remote Procedure Calls(RPC) named "Equip"[8]. It allows transparent data access to compliant equipment or computers by giving the equipment description. An Equip transaction is made between a client, generally in a console of the control room, and a server implemented on the equipment An RPC "Equip" server linked to 
FactoryLink as an external module has been written. Control rooms' consoles may now access the building safety processes as if it was home-made equipment.

- The CERN Central Alarm Server gathers alarms coming from a great number of systems all around the accelerators and dispatches them to the relevant consoles in the control rooms or to the firemen. A FactoryLink module has been written to monitor in an event driven way the building safety system's alarms and send them to the CAS using RPC.

\subsection{Remote Access}

Remote access to FactoryLink- may be given in two different ways:

- by using the facilities coming with the open-systems (X11, NFS, ...). This method might be used later in the control rooms

- by using a specific external $C$ module which we have designed to manage ASCII terminals and to display the database content through character based synoptics. It is thus possible to interact with FactoryLink through a simple telnet session. This feature is essentially used from desktop and home computers.

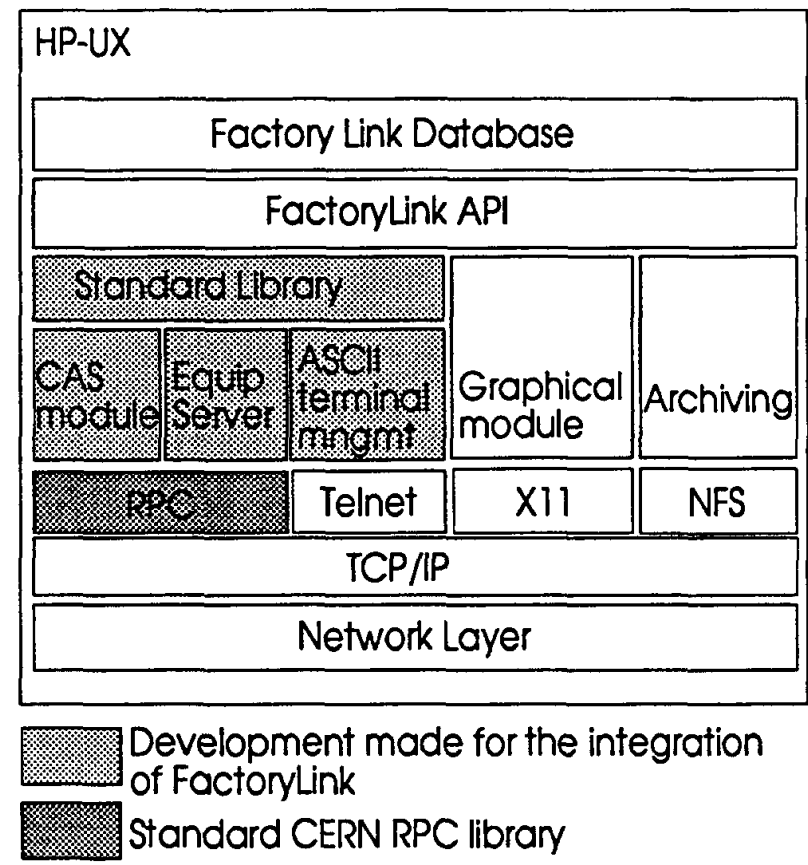

\section{Operational Experiences and Conclusion}

\subsection{Experiences with the Supervisor Itself}

FactoryLink proved to be stable and robust whilst highly demanding in computer resources because of the real-time constraints. It's industrial reliability makes no doubt. It is rather simple with it to develop and tune supervisory applications.

Some of the major advantages are the early availability of such applications in the installation process (at commissioning time) and the periodical enhancement due to new supervisory system releases.

\subsection{Experiences with the Integration}

The commitment of USDATA to open-systems made very easy the integration of FactoryLink in the CERN environment. In the installation process of industrial control systems we have set up an architecture and we have developed tools to smoothly and transparently integrate the two control systems. The different industrial processes, whilst still being autonomous and keeping their own characteristics(robustness, availability off-the-shelf), are seen as standard elements of the accelerator control system.

Figure 2: The Software Architecture

[1] Equipment Industrially Controlled

References

R.Saban, P.Ciriani, A. Guiard-Marigny, H.Laeger, M.Rabany and A.Swift, this conference (1993)

[2]Une librairie d'accès aux systèmes de supervision

F.Momal, D.Brahy and M.Dion, CERN AT Group Report 93-01 (IC) (1993)

[3]Une interface Sinec H1 entre FactoryLink et les automates Siemens

F.Momal and Ch. Clavier, CERN AT Group Report 93-02 (IC) (1993)

[4]Archivage de données d'automates Siemens

M.Dion, CERN AT Group Report 93-03 (IC) (1993)

[5]Une passerelle entre le monde des automates programmables industriels et le contrôle des accélérateurs

D.Brahy, CERN AT Group Report 93-05 (IC) (1993)

[6]FactoryLink IV Software System, User Manual

USDATA

[7]Interfacing Industrial Process Control Systems to LEP/LHC

M.Rabany, ICALEPS'91 Tsukuba, Japan - CERN AT Group Report 91-30 (CO) (1993)

[8]Accessing Equipment in the SPS-LEP Controls Infrastructure: The "SL Equip Package"

P.Charrue, CERN SL/9386 (CO) (1993) 
